\title{
PAIN IN THE RECTUM AND ANUS.
}

\author{
By RONALD W. RAVEN, F.R.C.S.
}

(Assistant Surgeon to the Gordon Hospital for Rectal Diseases and Surgical Registrar to the Royal Cancer Hospital.)

\section{INTRODUCTION.}

Pain is probably the most common symptom of lesions situated in the anorectal region. The anal canal is abundantly supplied with sensory nerves and lesions produce pain of varying severity. On the other hand, the sensory nerve supply of the rectum is poor and disease confined to this region may have progressed considerably before causing very much discomfort. This point is of importance in connection with malignant disease of the rectum-pain is not an early symptom.

When eliciting details from the patient concerning the symptom of pain in the ano-rectal region the following should be borne in mind. The date of onset and the location should be settled. An enquiry concerning the mode of onset, whether sudden or gradual, and the relation to defæcation, should be made. The character of the pain should be elicited from the patient as this may prove a clue concerning the causation. Pain in this region of the body may be continuous, interrupted, sharp, excruciating, burning, pricking, throbbing or bearing-down.

The importance of a careful clinical examination of a patient complaining of ano-rectal pain cannot be over-stressed. In the majority of patients a local lesion is present which may be obvious to the surgeon. On the other hand, the lesion may be easily overlooked. If no local cause for the pain is found after careful examination, attention should be given to other organs and especially the genitourinary system and the spinal cord.

It is proposed to discuss the commoner causes of pain in the ano-rectal region together with the appropriate treatment of the causative lesion. The various types of pain are correlated with the underlying cause; certain lesions give rise to a typical train of symptoms in almost every case. The subjects of hæmorrhoids and neoplasms are not dealt with in this paper.

\section{CORRELATION OF THE TYPE OF PAIN AND THE CAUSATIVE LESION.}

(a) Sharp pain during defæcation followed by severe drawing pain lasting for several hours.

The usual cause of this type of pain is a fissure-in-ano.

(b) Burning pain in the rectum.

The usual cause is proctitis.

(c) Continuous throbbing pain in the ano-rectal region.

This is generally indicative of peri-ano-rectal suppuration.

(d) Bearing-down pain in the rectum with a sense of fullness.

The usual cause of this symptom is either fæcal impaction, neoplasm, stricture of the rectum, foreign body, intussusception or enlargement of the prostate.

(e) Itching pain in the rectum or anus.

This phenomenon usually indicates threadworms, or peri-anal dermatitis. 
(f) Sensitive inflamed peri-anal skin.

Lesions producing an acrid discharge such as proctitis, fistula, malignant disease or a fissure are usually found.

(g) Pain in the sacro-coccygeal region.

Post-sacral dermoid cyst or tumour may be present.

(h) Paroxysmal or continuous drawing pain at the anus.

The usual causative lesion is either procidentia recti, hæmorrhoids or polypus.

(i) Sharp interrupted pain at the anus.

This may indicate external hæmorrhoids which may be thrombosed.

\section{PROCTITIS.}

The importance of this condition is often overlooked except by the proctologist who recognizes its seriousness to the patient. It may give rise to peri-rectal suppuration, fistulæ, stricture of the rectum or extensive ulceration. The inflammatory process may be limited to a part of the rectum or the whole organ may be involved. It may involve the bowel above for varying distances. In certain cases the inflammation does not extend deeper than the mucous membrane; in others the deeper tissues are involved and even the peri-rectal structures do not escape.

\section{(a) Acute catarrhal proctitis.}

In children this condition may be a complication occurring during the course of a specific fever. In adults the cause may be ascribable to excessive purgation, parasitic infection, constipation or chemical irritants.

Symptoms. The patient experiences burning pain in the rectum of varying intensity. The peri-anal skin may be sensitive and inflamed. There is desire for frequent evacuation of the rectum with tenesmus. The stools are loose and contain an excessive amount of mucus and varying quantities of blood.

Signs. On examination of the rectum with the proctoscope the mucous membrane is found to be congested and odematous. The whole of the rectum may be involved or only a circumscribed area. The mucous membrane is either bright or dark red and it may have a granular appearance. There is an excessive amount of mucus and the fæces are watery and bloodstained. In a few cases a diphtheritic-like membrane is present.

Treatment. It is preferable to have the patient in bed. The diet in the main should be fluid. The rectum is cleansed twice daily with an antiseptic or astringent lotion. The writer has found a 2 per cent. solution of mercurochrome of value. Others advocate potassium permanganate, boric acid, argyrol, silver nitrate or ichthyol. Pain is relieved by suppositories containing morphine or cocaine. Excoriation of the peri-anal skin is prevented by the application of zinc oxide ointment.

Surgical intervention is necessary if complications such as fistula or perirectal suppuration occur.

\section{(b) Chronic Catarrhal Proctitis.}

The organism reponsible for this condition has not been isolated. There are numerous factors which may be responsible for chronic catarrhal proctitis 
amongst which mention is made of the following. Infection in the gastrointestinal tract and especially in the appendix may be the main contributory factor. The condition may be secondary to malignant disease of the abdominal or pelvic organs. Any condition leading to congestion of the rectum or to an irritating discharge may cause chronic proctitis. The lesion may be associated with psychological or neurogenic disturbances.

Symptoms. If the disease is an accompaniment of catarrhal colitis the symptoms associated with the latter condition are manifested. In patients where the inflammation is limited to the rectum there is burning pain of varying severity, a sense of fullness in the rectum, tenesmus and aching pain in the sacro-coccygeal region. The stools are liquid and contain muco-pus and blood.

Signs. There is generally excoriation and redness of the peri-anal skin and superficial fissures may be present. The mucous membrane of the rectum is reddened, thickened and erosions may be present. There is excessive mucopurulent secretion.

In certain cases the mucous membrane of the rectum has a granular appearance and papillomata may be seen. This is the hypertrophic type.

In other cases the mucosa is dry and fissured. Particles of fæcal matter are adherent to the rectal wall together with dried mucus. This has been described as the atrophic type.

Treatment. The patient should be placed in hygienic surroundings and the diet must be nutritious excluding coarse vegetables, condiments and alcohol. If anæmia is present iron must be given. Bismuth, thymol or salol are useful in combating intestinal putrefaction and lessening diarrhœe. Opiates may be required in order to relieve the rectal pain and tenesmus and morphia should therefore be given as required. Suppositories containing morphia and belladonna are valuable adjuncts. Colonic lavage serves to evacuate irritating scybalæ and mucous discharge. Local applications of 2 per cent. mercurochrome to the mucous membrane of the rectum are of use. If hæmorrhoids, adenomata, polyps, fissures or fistula be present, surgical intervention is indicated for their removal as the proctitis generally persists until all sources of irritation are removed.

\section{ANAL FISSURE.}

This condition is one of the commonest causes of pain in the ano-rectal region. No lesion of similar size in the gastro-intestinal system produces so much pain and disability. The pain is experienced with varying degrees of severity during defæcation and is followed by severe drawing pain immediately after defæcation. Aching or throbbing pain may persist for several hours afterwards. A fissure may be found at any point in the anal ring but usually at or near the posterior commissure of the anal canal. In the early stages the ulcer is superficial and elliptical in shape, the margins are inverted and non-indurated. If the condition remains untreated, local infection supervenes and the ulcer becomes hard and indurated at its edges, which become undermined. A "sentinel pile" is frequently seen at the lower margin of the fissure. In long-standing cases pathological changes occur in the sphincteric muscles. Fibrosis occurs within the muscle bundles and causes loss of elasticity leading to a contracted anus. 


\section{Treatment.}

r. In the Early Stages. It is possible to effect healing of a recent superficial fissure by means of the local application of such substances as silver nitrate as a stick or pure ichthyol applied on a wooden probe. The application of analgesic ointment will relieve the pain in these cases. Ointments containing percaine or anæsthesin are usually effective and they may be applied digitally. If the granulation tissue covering the base of the ulcer requires stimulation then the application of lotio rubra or scarlet-red ointment is indicated.

2. Cases with Sphincteric Spasm. In cases of early fissure with spasm of the sphincter muscle the injection of an anæsthetic solution in oil will often be effective.

A solution which the writer has found very valuable is "A.B.A.". This is made up in ampoules of 5 c.c by Messrs. Allen \& Hanburys and consists of a 3 per cent. solution of anæsthesin with 5 per cent. benzyl alcohol and Io per cent. ether in sterilized olive oil.

Method of injection. The patient is placed in the left lateral position and the peri-anal skin is prepared by painting with tincture of iodine. The ampoule of "A.B.A" is warmed in hot water and a ro c.c Record syringe is filled. The right index finger is inserted in the rectum and the needle of the syringe enters the tissues at a point I inch behind the anus in the middle line. The "A.B.A." is injected into the sphincter on each side of the mid-line in equal amounts. Finally about $\frac{1}{2}$ c.c. is injected deeply to the fissure. It is essential that the patient's bowels are regulated and it is desirable for the stools to be soft. Senna and liquid paraffin are useful agents for this. purpose. If the stools are hard and constipated, olive oil enemata are of value.

3. Chronic Anal Fissure. In the chronic stage an anal fissure presents an indurated edge which is easily palpable on digital examination and a "sentinel pile" is usually present. In certain cases other pathological conditions of the rectum and anus are present. Surgical operation is essential for permanent cure in this stage. There are two surgical procedures advocated for the cure of a chronic anal fissure.

(a) Miles' operation-Pectenotomy. In cases of anal fissure the pecten band is always well marked. This consists of a deposit of fibrous tissue in the submucosa of the region of the anal canal between Hilton's white line and the edges of the Morgagnian valves. The pecten band interferes with the normal expansion of the anal orifice during defæcation. The efforts of the levatores ani muscles to expand the anus cause reflex spasm of the external sphincter muscle. After division of the pecten band the fissure is no longer subjected to the effects of external sphincter spasm and cicatrisation occurs.

The operation may be performed under a low spinal anæsthesia with the patient in the right lateral semi-prone position. The usual toilette of the anus is carried out and the lower half of the anal canal is everted by the introduction of the left index finger as far as the distal interphalangeal joint and flexing the finger forcibly. The skin of the anus is pressed outwards by the left thumb at the same time in order to push the inner border of the external sphincter outwards. In the right posterior quadrant an incision is made through the mucosa 
of the lower part of the anal canal and the pecten band is seen encircling the anus as a white band. The pecten band is then carefully divided. To prevent early healing of the wound a small piece of cotton wool soaked in mercury perchloride ( $I$ in 500 ) is inserted.

For the post-operative pain, which may be severe, morphia should be given. The dressings are changed after twenty-four hours and the anus cleansed. It is necessary to change the dressings twice each day and after each action of the bowels. The pledget of cotton wool is removed from the wound on the fourth day. The bowels are opened on the fifth day and the patient remains in bed for fourteen days. In Miles' experience this operation has never failed to cure an anal fissure.

(b) Gabriel's operation. The objects of this operation are to secure external drainage of the fissure and to overcome a spastic or contracted external sphincter.

The operation is performed under low spinal anæsthesia and the patient placed in the lithotomy position. The anus is prepared in the usual manner and a triangular incision is made on each side of the fissure commencing at the anal margin. The lateral incisions are two inches long and are joined at their posterior extremities by a transverse incision. This triangular area of skin is dissected up and the fissure together with sentinel pile are excised. If a fibrous band constricts the anal canal it is divided. The external wound is cauterized with the diathermy electrode to prevent the margins of the external wound adhering together. Vaseline is injected into the rectum, a tube inserted and dressings applied. A bougie is passed into the rectum twice a day after the third day. This is continued for several weeks at lengthening intervals.

\section{PRURITUS ANI.}

This term indicates a definite pathological condition of the skin of the anus and peri-anal tissues. The condition is peri-anal dermatitis and may be acute or chronic. In the acute stage the skin is red and excoriated and the peri-anal tissues are odematous. In the chronic variety the skin is thickened, indurated and has lost its normal elasticity. The chief symptom complained of is intense itching pain of the anus and surrounding tissues. This pain may be worse during the night, causing severe loss of sleep leading to chronic ill-health.

\section{Some Etiological Factors.}

(a). Pathological Conditions of the Anus and Rectum. In the majority of patients a co-existent lesion of the anus or rectum is found as the cause of the pruritus. Thus the following conditions must be eliminated:-pectinosis, fissurein-ano, fistula, cryptitis, proctitis, prolapsing internal hæmorrhoids, prolapse of the mucous membrane of the rectum.

(b). Pathological Condition of the Female Genital Organs. Any condition of the female genital organs which gives rise to a vaginal discharge must be treated. Such vaginal discharges act as local irritants.

(c). Parasites. Intestinal worms may cause an itching pain within the anal canal or at the anal orifice when they lie concealed between folds of mucous membrane or folds of thickened peri-anal skin. In children oxyuris vermicularis is found in the cæcum and rectum. In adults thread worms are fairly common in the large intestine and rectum. 
(d). Drugs. The ingestion of certain drugs sometimes induces severe pruritus which may be generalized or localized to the perineum. Common examples of these drugs are morphine, cocaine, quinine and belladonna.

(e). Certain Diseases. In metabolic disorders such as diabetes mellitus and gout, toxic products may cause pruritus. In glycosuria the urine acts as a local irritant. Portal congestion due to disease of the liver may give rise to pruritus ani.

\section{Treatment.}

If a local lesion in the ano-rectal region is present this must be treated. Thus fissures, fistulæ and hæmorrhoids must be dealt with in the first instance.

Constipation should be avoided and careful anal hygiene carried out after defæcation.

A large number of local applications have been advocated and it is often necessary to experiment with several lotions or ointments until one is found which brings relief. A useful lotion is composed of equal parts of 2 per cent. malachite green in 80 per cent. alcohol and 2 per cent. mercuric chloride in 80 per cent. alcohol. The peri-anal skin is painted twice daily with this lotion. In certain cases an ointment containing I per cent. percaine is of value. Another lotion of value is lotio calaminæ containing 5 per cent. carbolic acid. In patients with œedema of the peri-anal tissues a lotion of lead lactate often gives reliefI dramme of liquor plumbi subacetatis is mixed with 7 drammes of fresh milk.

In the presence of acute peri-anal dermatitis an ointment consisting of the following should be applied:-

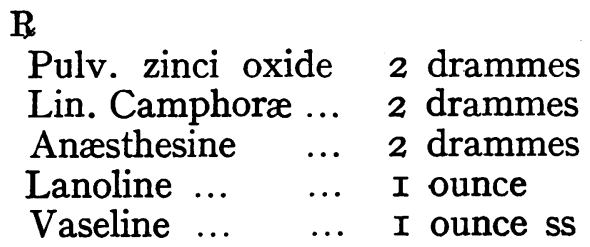

In obstinate cases of pruritus ani a saturated solution of silver nitrate is useful. The solution is applied and left for ten minutes, after which a solution of sodium chloride-strength I dramme to I pint-is applied in order to neutralize the silver nitrate.

Injection Therapy. Solutions have been injected into the peri-anal tissues in cases of pruritus in order to produce certain cutaneous anæsthesia. Good results in many cases of obstinate pruritus ani have been obtained after the injection of a solution known as "A.B.A." which is composed of 3 per cent. anæsthesine, 5 per cent. benzyl alcohol and Io per cent. ether, made up in sterile olive oil. It is advisable to give a few injections of a large amount of A.B.A. rather than a series of injections of a small quantity. On three occasions at weekly intervals Io cc. of A.B.A. are injected into the circumference of the perianal tissues.

X-ray Therapy. In many cases exhibiting obstinate pruritus ani marked improvement has occurred after X-ray therapy. The dose usually employed is one-third of an erythema dose, preferably unfiltered, from a gas tube and repeated after an interval of fourteen days. If necessary a third dose may be given after a further interval of one month. 
Operative Treatment. In intractable pruritus ani it may be necessary finally to resort to operative surgery. Recently an operation found to give good results is division of certain fibres of the perineal nerve. Ball's operation, involving undercutting of the anal and peri-anal skin in order to section the sensory nerve supply gives unsatisfactory results.

\section{RECTAL PAIN AS A SYMPTOM OF DISEASES OF OTHER ORGANS.}

(a). Disease of the Uterus or Appendages. Disease of organs in close proximity to the rectum may cause rectal pain. If the rectum is found to be healthy in patients complaining of rectal pain, it is essential that the uterus and appendages should be investigated. In such cases displacements of the uterus may be found and when rectified the rectal pain will disappear. Enlargement of the uterus due to fibroids or malignant disease sometimes causes pain in the rectum. In other cases displacement of the ovary or chronic salpingo-oophoritis may be found.

(b). Disease of the Prostate. Enlargement of the prostate and especially the hypertrophic variety often gives rise to discomfort or pain in rectum and anus.

(c). Disease of the Urinary Bladder. Pain may be referred to the rectum from lesions occurring in the urinary bladder. Thus cystitis or carcinoma may be discovered as the cause.

(d). Disease of the Spinal Cord. In any patient with rectal pain, if there is no cause to be found in the rectum or other pelvic organs, the central nervous system should be investigated. In rare instances rectal pain is found to be one of the earliest manifestations of disseminated sclerosis. In tabes dorsalis pain in the rectum may be so severe as to constitute rectal crises. This is accompanied by distressing tenesmus and the sensation caused by a foreign body in the rectum. In these cases other manifestations of tertiary syphilis may be present.

\section{RECTAL NEUROSIS.}

Patients exhibiting true rectal neurosis are rare. The proctologist can find no abnormality in the ano-rectal region and the aid of the psychologist is usually invoked. The pain is very real to these patients and may occur in excrutiating paroxysms. The greatest care and sympathy must be extended to these unfortunate people as several have been driven to acts of desperation.

\section{RECTAL NEURITIS.}

Inflammation of certain nerves supplying the rectum is sometimes encountered as a cause of rectal pain. Sometimes this is accompanied by sciatica. Patients with rectal neuritis complain of sharp severe pain referred to the anus and rectum and this is often accentuated at night and after defæcation. 